\title{
A brief introduction to biophotonic techniques and methods
}

\author{
Qingming Luo ${ }^{1,2^{*}}$ \\ ${ }^{1}$ School of Biomedical Engineering, Hainan University, Haikou 570228, China; \\ ${ }^{2}$ Britton Chance Center for Biomedical Photonics, MoE Key Laboratory for Biomedical Photonics, Wuhan National Laboratory for \\ Optoelectronics-Huazhong University of Science and Technology, Wuhan 430074, China
}

Received November 24, 2020; published online December 1, 2020

Citation: Luo, Q. (2020). A brief introduction to biophotonic techniques and methods. Sci China Life Sci 63, 1771-1775. https://doi.org/10.1007/s11427-020$1858-1$

Biophotonics is not a standard word listed in a classical dictionary. The meaning of "biophotonics" is essentially the use of photonic technologies to detect and perhaps to control a biological phenomenon or event, at different levels ranging from molecules, cells, tissue, organisms, to intact animals and even human bodies (Yeh and Krishnan, 2018). The field of biophotonics is not well defined because it is a multidisciplinary research field at the intersection of physics, biology, chemistry, medicine and engineering (Tsia, 2016; Vo-Dinh, 2014; Wang and Tuchin, 2016). As alternatives to biophotonics, the term "biomedical photonics" and "biomedical optics" have also been popularly used, with no exact distinction among them (Vo-Dinh, 2014).

On the other hand, the precise meanings of the term optics and photonics are not clear, at least to some extent. Some researchers think that photonics include topics that rely on the interaction of light and matter, while optics is used to study light propagation in both free-space and medium (Saleh and Teich, 2019). Other researchers may consider photonics as a field that involves the energy from the entire electromagnetic spectrum, while optics deals only with a particular type of light radiation that can be seen by the human eye (Vo-Dinh, 2014). After considering the multidisciplinary nature of biophotonics, we tend to agree with the former opinion, because it provides more room and opportunities for the blooming of biophotonics.

The field of Biophotonics probably began with two Dutch

\footnotetext{
*Corresponding author (email: qluo@hainanu.edu.cn)
}

spectacle makers, Hans Jansen and his son Zacharias, who were recognized as the inventors of the first compound microscope in 1595; however, they left no publications describing any observations from their microscope (https:// www.nature.com/milestones/milelight/timeline.html). Seventy years later, in 1665, Robert Hooke, who was a Fellow of the Royal Society in London and thus truly a scientist, published a historic book called Micrographia, where he described the modern microscope he refined, including a stage, a light source and three optical lenses, and illustrated many beautiful structures he observed using the microscope. Micrographia demonstrated for the first time what light microscopy could do for science, and inspired a wide public interest in the field of light microscopy (https://www.nature. $\mathrm{com} / \mathrm{milestones/milelight/timeline.html).} \mathrm{However,} \mathrm{due} \mathrm{to}$ the spherical and chromatic aberration in the compound microscope, many researchers at that time preferred to use simple microscope before achromatic lenses were widely used in the early 19th century (https://www.nature.com/ milestones/milelight/timeline.html). And, because simple microscope could resolve detail as small as $1 \mathrm{~mm}$ using only a tiny convex lens, they had been popularly used in parallel with compound microscope for a long period of time. Interestingly, although not the first creator of microscopes, the Dutch draper Antonie van Leeuwenhoek constructed more than 500 simple microscopes, which laid the foundation of modern cell biology (Croft, 2006).

Another milestone that nobody would ignore in the early history of light microscopy is the publication of a landmark 
paper by Ernst Abbe in 1873. Although no mathematical equations actually appeared in this paper, Abbe reported there the resolution limit of light microscopy - half the wavelength of the imaging light. This limit has been widely accepted for a long time until the invention of super-resolution microscopy in the early 2000s. In this and the following papers, Abbe provided quantitative insights into the image formation and lens aberrations in light microscopy, which pushed forward the proper design of microscope lenses. In collaboration with Carl Zeiss and Otto Schott in Jena, Abbe also made systematic improvements in the production of commercial light microscope in the 19th century (https://www.nature.com/milestones/milelight/timeline. html).

Improving the image contrast of light microscopy (especially bright-field microscopy) has been a long-lasting task. Although Robert Hooke and many others reported their accidental observations on the appearance of a stained object under a light microscope, Joseph von Gerlach deserves credit for recognizing the power of differential staining and for describing his staining method carefully in 1858 (https:// www.nature.com/milestones/milelight/timeline.html). And, the development and use of new stains and synthetic dyes in the late 1800s and early 1900s significantly pushed forward the image contrast of bright-field microscopy, and enabled the discovery of many cytological phenomena. For example, the use of silver staining in cytology, pioneered by Camillo Golgi and popularized by Santiago Ramón y Cajal, opened new opportunities in neuroanatomical observations, while the haematoxylin and eosin stain developed by Paul Mayer in 1896 became a key diagnostic method in current clinical laboratory (https://www.nature.com/milestones/milelight/ timeline.html).

Scientists had been trying many other ways to improve the image contrast of light microscopy (https://www.nature. com/milestones/milelight/timeline.html). The physicist Oskar Heimstadt successfully constructed the first fluorescence microscope in 1911, shortly after the observation by August Kohler who, in 1904, found that some objects emitted light of longer wavelength when illuminated with UV light. In 1914, Stanislav von Provazek reported the first use of a fluorescent dye as a stain to enhance the autofluorescence of cells and tissues. The German pharmacologist Philipp Ellinger and anatomist August Hirt invented the first epifluorescence microscope in 1929, and used it to image kidney and liver tissue in rodents injected with fluorescent dyes. In 1942, Albert Coons and coworkers successfully obtained bright fluorescent antibodies without disturbing their antigen-binding properties, which started the era of immunofluorescence microscopy (https://www.nature.com/ milestones/milelight/timeline.html).

Fluorescence could provide infinite contrast if the right imaging conditions are used. With the invention of dichro- matic mirrors in 1967, and more importantly the lasers in 1960s as the powerful light source, many advanced fluorescence microscopy techniques had been invented and/ or upgraded (https://www.nature.com/milestones/milelight/ timeline.html), including confocal microscopy (constructed in 1955 (Croft, 2006), patented in 1957 (Pawley, 2013), granted in 1961 (https://www.nature.com/milestones/milelight/timeline.html)), Forster (or fluorescence) resonance energy transfer microscopy in 1976, total internal reflection fluorescence microscopy (TIRF) in 1981, deconvolution microscopy in 1983, two-photon microscopy in 1990, light sheet microscopy and near-field scanning optical microscopy in 1993, as well as the successful implementation of stimulated emission-depletion (STED) microscope in 2000. Note that STED opens a new era of super-resolution microscopy. In addition to the invention of various fluorescence microscopy techniques, other approaches not relying on fluorescence as the contrast were also invented and popularly used, including but not limited to phase contrast microscopy in 1935 , polarization microscopy in 1939 , and differential interference contrast microscopy in 1955 (https://www.nature. $\mathrm{com} / \mathrm{milestones} / \mathrm{milelight/timeline.html)}$.

It should be mentioned that many useful technologies developed for other applications have significantly pushed the advances of light microscopy by providing upgrades for its components. The most famous examples include the lasers for the light source, the charge-coupled device (CCD) cameras for the detector, the micro-electronic circuitries (microchips) for system control, and the computer for the design and manufacture of optics and image processing (Vo-Dinh, 2014). Furthermore, although the principle and basic design of light microscopy has remained almost constant for more than a century (Piston, 2009), the performance of various light microscopy techniques has been steadily improved in response to the practical needs from different application fields. For example, to study the structure-function relationship of the nervous system, which is important in the field of neuroscience, a large amount of innovations in biophotonics have been proposed (Lichtman and Denk, 2011). One amazing example is the development of calcium imaging technique for detecting a fast event called action potential. Action potential is an explosion of electrical activity that sends out information from one part of a neuron to a distant site, and direct detection of action potentials from many neuron cells simultaneously would be a big challenge because action potentials occur typically only in several milliseconds (Grienberger and Konnerth, 2012). After the continuous efforts in the development of genetically encoded calcium indicators (Dana et al., 2016; Lin and Schnitzer, 2016) and in vivo two-photon microscopy (Cheng et al., 2011; Sofroniew et al., 2016), calcium imaging can be now routinely performed in vivo and in awake animals. Intensive efforts have also been made to solve another big challenge in 
neuroscience-mapping neural connectivity. It is known that synaptic connections among neurons are distributed through branches in two important components called axons and dendrites, and the length of all the branches of a neuron (for example, pyramidal cell) may exceed a centimeter in a mouse (Lichtman and Denk, 2011). If one wants to figure out the $3 \mathrm{D}$ projection of a neuron in a mouse brain, the imaging would need to be performed at very high resolution (to identify all the fine branches) and a large sample size (to collect all branches). Luo and his team have been focusing on whole-brain optical imaging technologies for more than 10 years, and finally developed a complete technical system for visualizing brain-wide networks of neuronal connectivity (Li et al., 2010; Shi and Luo, 2017). Recently, by combining fluorescence micro-optical sectioning tomography (MOST) technique with monosynaptic rabies viral tracers, they mapped long-range inputs to GABAergic interneurons in the mouse medial prefrontal cortex (Sun et al., 2019).

Here we present a brief survey of the history and some important milestones in light microscopy, which is one of the most valuable biophotonic techniques; however, we should remember that biophotonic technologies and methods include not only light microscopy. However, due to the limited space in this special topic, we do not have a chance to give a survey of other important biophotonic techniques, including but not limited to spectroscopy, sensing and manipulation (Di Bartolo and Collins, 2010; Keiser, 2016; Tuchin, 2010), which have surely made a major impact on biophotonics. To give a sense of such an impact, optogenetics, a method that combines optics and genetics to control the activities of cells or even live animals, completely changes the way neuroscientists think about biophotonic manipulation (Fenno et al., 2011; Goglia and Toettcher, 2019; Krueger et al., 2019). Another great example is optical tweezers, which use optical forces to provide a precise manipulation and measurement on the forces and displacements of small particles and macromolecules in live cells (Fazal and Block, 2011; Killian et al., 2018; Moffitt et al., 2008), or even controlling cells within living animals (Zhong et al., 2013). Note that the work of optical tweezers have been awarded the Nobel Prize of Physics twice (1997 and 2018). Regarding the clinical applications of biophotonic techniques, optical coherence tomography (OCT) has become the golden standard in ophthalmology by providing high-resolution 3D images of the retina, the retinal nerve fiber layer and the optic nerve head, which help to detect and manage retinal diseases (Adhi and Duker, 2013; Popp et al., 2012). Additionally, although sometimes Biophotonics is considered to cover only applications in life sciences and fundamental biomedical research, the term Biophotonics seems to have evolved historically, and now even reaches into application fields in everyday life, for example, food and pharmaceutical analysis.

In the last year, we selectively invited several Chinese research groups to contribute to this special topic of Science China Life Sciences. Now we have a collection of one research article and four review articles to show new findings or understandings on the biophotonic techniques and methods. We expect this collection of papers will represent, at least to some extent, the current multidisciplinary research of biophotonics in China. For an introduction to the research groups in the biophotonics field in China and their representative contributions, we recommend our previous review (Shi and Luo, 2017).

The research paper by Tingwei Quan and coworkers aimed to solve a data processing problem in calcium imaging. It is generally believed that calcium imaging is a powerful technique for measuring the functional activity of neural populations. In this technique, a threshold is necessary to transform the fluorescence signals from neurons into action potential (AP) trains, and the reconstruction accuracy is determined by the threshold selection. However, it is hard to find an optimal threshold. Tingwei Quan and coworkers (Shen et al., 2020) proposed a new reconstruction method that is based on the prior shape of the AP train and calcium signal baseline. In their method, the reconstructed AP train can be directly obtained without a threshold operation, which makes the reconstruction robust to the baseline fluctuations of the calcium signal. They verified the performance of the new method using experimental and simulation calcium signals.

The review paper given by Prof. Zhen-Li Huang and his group members aimed to provide an updated understanding on how to estimate the resolution of fluorescence microscopy. Resolution is undoubtedly the most important parameter in light microscopy. However, with the blooming of various advanced fluorescence microscopy techniques, especially after the invention of super-resolution fluorescence microscopy in the past two decades, many methods have been proposed for estimating the resolution of fluorescence microscopy, and researchers who are not experts in light microscopy would sometimes be confused on which method they should use for their specific experimental conditions. The review paper by Zhen-Li Huang et al. (Li and Huang, 2020) answered this question in a timely manner. They discussed the theoretical and practical issues regarding the resolution estimation in fluorescence microscopy.

Another review by Hangrong Chen and collaborators (Xue et al., 2020) discussed the design strategy of optical probes for tumor hypoxia imaging. Solid tumors usually form a complex microenvironment to promote the transformation of normal cells into cancerous ones, and hypoxia is a common feature of most solid tumors. The design and use of optical probes for imaging hypoxia sites and monitoring tumor oxygen levels has been a hot topic in precision tumor therapy. This review discusses two most promising strategies to detect tumor hypoxia: oxygen probes for detecting the hy- 
poxia level directly, or other probes (NTR- and AzoR-responsive hypoxia probes) for detecting hypoxia-assisted reduced microenvironments. This review is informative for researchers who are interested in designing hypoxia imaging probes or looking for new treatment practice of hypoxic tumors.

Prof. Sung-Liang Chen and coworkers (Seong and Chen, 2020) presented a review paper discussing a special technique called photoacoustic microscopy (PAM), which belongs to the field of photoacoustic imaging. PAM has promising potentials in clinical use and has received great attention in the applications of human tissues. In this review, Chen et al. discussed the principle of PAM and its applications to different kinds of human tissues, including breast tissue, eyes, gastrointestinal tissue, and many others. They pointed out that further technology developments and clinical studies would turn PAM into a promising biophotonic technique for clinical use.

In the last review, Qiong $\mathrm{Wu}$ and the team members ( $\mathrm{Ji}$ et al., 2020) discussed a special kind of biomaterial called photosensitive hydrogels, which can undergo physical or chemical changes in response to an optical signal and thus are regarded as an ideal candidate for use in fields such as biological materials, drug carriers, and sensors. In this review, $\mathrm{Wu}$ et al. summarized the structure, photochemical mechanisms and the preparation of photosensitive hydrogels, explored the various bio-applications of photosensitive hydrogels, and described the potential needs in synthesizing more sophisticated hydrogels with special photophysical properties.

Finally, when looking into the future of biophotonics, we would like to emphasize that biophotonics is a multidisciplinary field that is gathered with not only scientists who are interested mainly in understanding nature, but also engineers and physicians who have great enthusiasms to respond to the needs in our daily lives. Actually, in response to the COVID-19 pandemic, a joint group from Spain, Israel, and the United Kingdom has recently presented a perspective paper discussing how to use UV-C light as an affordable way to limit the transmission of SARS-CoV-2 in indoor spaces (García de Abajo et al., 2020). We surely are willing to see more work like this in the near future.

Compliance and ethics The author(s) declare that they have no conflict of interest.

\section{References}

Adhi, M., and Duker, J.S. (2013). Optical coherence tomography—current and future applications. Curr Opin Ophthalmol 24, 213-221.

Cheng, A., Gonçalves, J.T., Golshani, P., Arisaka, K., and Portera-Cailliau, C. (2011). Simultaneous two-photon calcium imaging at different depths with spatiotemporal multiplexing. Nat Methods 8, 139-142.

Croft, W.J. (2006). Under the Microscope: A Brief History of Microscopy
(Singapore: World Scientific).

Dana, H., Mohar, B., Sun, Y., Narayan, S., Gordus, A., Hasseman, J.P., Tsegaye, G., Holt, G.T., Hu, A., Walpita, D., et al. (2016). Sensitive red protein calcium indicators for imaging neural activity. eLife 5, e12727.

García de Abajo, F.J., Hernández, R.J., Kaminer, I., Meyerhans, A., RosellLlompart, J., and Sanchez-Elsner, T. (2020). Back to normal: an old physics route to reduce SARS-CoV-2 transmission in indoor spaces. ACS Nano 14, 7704-7713.

Di Bartolo, B., and Collins, J. (2010). Biophotonics: Spectroscopy, Imaging, Sensing, and Manipulation (Springer, The Netherlands).

Fazal, F.M., and Block, S.M. (2011). Optical tweezers study life under tension. Nat Photon 5, 318-321.

Fenno, L., Yizhar, O., and Deisseroth, K. (2011). The development and application of optogenetics. Annu Rev Neurosci 34, 389-412.

Goglia, A.G., and Toettcher, J.E. (2019). A bright future: optogenetics to dissect the spatiotemporal control of cell behavior. Curr Opin Chem Biol 48, 106-113.

Grienberger, C., and Konnerth, A. (2012). Imaging calcium in neurons. Neuron 73, 862-885.

Ji, W., Wu, Q.W., Han, X., Zhang, W., Wei, W., Chen, L., Li, L., and Huang, W. (2020). Photosensitive hydrogels: from structure, mechanisms, design to bioapplications. Sci China Life Sci 63, 1813-1828.

Killian, J.L., Ye, F., and Wang, M.D. (2018). Optical tweezers: a force to be reckoned with. Cell 175, 1445-1448.

Krueger, D., Izquierdo, E., Viswanathan, R., Hartmann, J., Pallares Cartes, C., and De Renzis, S. (2019). Principles and applications of optogenetics in developmental biology. Development 146, dev175067.

Li, A., Gong, H., Zhang, B., Wang, Q., Yan, C., Wu, J., Liu, Q., Zeng, S., and Luo, Q. (2010). Micro-optical sectioning tomography to obtain a high-resolution atlas of the mouse brain. Science 330, 1404-1408.

Li, M., and Huang, Z.L. (2020). Rethinking resolution estimation in fluorescence microscopy: from theoretical resolution criteria to superresolution microscopy. Sci China Life Sci 63, 1776-1785.

Lichtman, J.W., and Denk, W. (2011). The big and the small: challenges of imaging the brain's circuits. Science 334, 618-623.

Lin, M.Z., and Schnitzer, M.J. (2016). Genetically encoded indicators of neuronal activity. Nat Neurosci 19, 1142-1153.

Moffitt, J.R., Chemla, Y.R., Smith, S.B., and Bustamante, C. (2008). Recent advances in optical tweezers. Annu Rev Biochem 77, 205-228.

Pawley, J. (2013). Handbook of Biological Confocal Microscopy (Springer US).

Piston, D.W. (2009). The impact of technology on light microscopy. Nat Cell Biol 11, S23-S24.

Popp, J., Tuchin, V.V., Chiou, A., and Heinemann, S.H. (2012). Handbook of Biophotonics, Vol. 1: Basics and Techniques (Weinheim: WileyVCH Verlag).

Saleh, B.E.A., and Teich, M.C. (2019). Fundamentals of Photonics (Hoboken: John Wiley \& Sons).

Seong, M., and Chen, S.L. (2020). Recent advances toward clinical applications of photoacoustic microscopy: a review. Sci China Life Sci 63, 1798-1812.

Shen, Y., Quan, T., Wang, M., Liu, X., Lv, X., Chen, X., and Zeng, S. (2020). Neural spike train reconstruction from calcium imaging via signal-shape composition model. Sci China Life Sci 63, 1829-1832.

Shi, H., and Luo, Q. (2017). Biophotonics in China. J Biophoton 10, 15721579.

Sofroniew, N.J., Flickinger, D., King, J., and Svoboda, K. (2016). A large field of view two-photon mesoscope with subcellular resolution for in vivo imaging. eLife 5, e14472.

Sun, Q., Li, X., Ren, M., Zhao, M., Zhong, Q., Ren, Y., Luo, P., Ni, H., Zhang, X., Zhang, C., et al. (2019). A whole-brain map of long-range inputs to GABAergic interneurons in the mouse medial prefrontal cortex. Nat Neurosci 22, 1357-1370.

Tsia, K. (2016). Understanding Biophotonics: Fundamentals, Advances, and Applications (Boca Raton: Jenny Stanford Publishing).

Tuchin, V.V. (2010). Handbook of Photonics for Biomedical Science (Boca Raton: CRC Press). 
Vo-Dinh, T. (2014). Biomedical Photonics Handbook, Second Edition, Volume I, Fundamentals, Devices, and Techniques (Boca Raton: CRC Press).

Wang, R.K., and Tuchin, V.V. (2016). Advanced Biophotonics: Tissue Optical Sectioning (Boca Raton: CRC Press).

Xue, F., Chen, J., and Chen, H. (2020). Design strategy of optical probes for tumor hypoxia imaging. Sci China Life Sci 63, 1786-1797.

Yeh, Y., and Krishnan, V.V. (2018). Biophotonics: Science and Technology (Singapore: World Scientific).

Zhong, M.C., Wei, X.B., Zhou, J.H., Wang, Z.Q., and Li, Y.M. (2013). Trapping red blood cells in living animals using optical tweezers. Nat Commun 4, 1768.

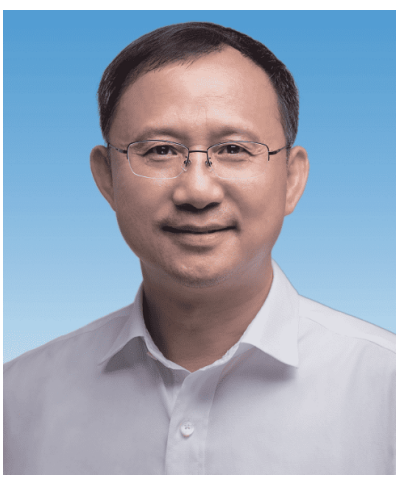

\section{Biographical Sketch}

Qingming Luo received his B.S. degree in Technical Physics from Xidian University, Xi'an, China, M.S. degree in optics and Ph.D. in physicoelectronics and optoelectronics from Huazhong University of Science and Technology (HUST), Wuhan, China. He spent one and a half year as a Postdoctoral Research Associate in the Department of Biochemistry and Biophysics, University of Pennsylvania, Philadelphia, USA, working with the late biomedical photonics pioneer Britton Chance. After returning to HUST in 1997, he founded the first lab for biomedical photonics in China, and became the first Cheung Kong Distinguished Professor of biomedical photonics recognized by Ministry of Education of China (MoE). Luo's lab has been approved as the Key Laboratory of Biomedical Photonics of MoE since 2000. He was appointed as the Executive Deputy Director of Wuhan National Laboratory for Optoelectronics (WNLO) in 2007. After more than 10 years' efforts, WNLO has become a worldfamous research center in the field of optics, photonics, and optoelectronics, as well as the core engine of Wuhan Optics Valley of China. Dr. Luo was then appointed by Ministry of Science and Technology of China as the Director of WNLO in 2017. After 11 years' serving as the Vice President of HUST, Dr. Luo was appointed by Hainan province as the President of Hainan University in 2018.

Dr. Luo's research interests focus on multi-scale optical bioimaging and cross-level information integration. He is an elected Member of the Chinese Academy of Sciences, elected Fellow of The International Academy of Medical and Biological Engineering (IAMBE), The American Institute for Medical and Biological Engineering (AIMBE), The International Society for Optics and Photonics (SPIE), The Institution of Engineering and Technology (IET), The Optical Society (OSA), and Chinese Optical Society (COS). With his leading contributions, the Biomedical Engineering in HUST was rated A+ in the latest 4th round of China Discipline Ranking. Dr. Luo is the elected Chair of Biomedical Engineering Steering Committee for Guidance in Teaching in Higher Educations Institutions 2018-2022 appointed by MoE. 